\title{
The Study of Epidemiology and Etiological Factors for Pregnancy Related Acute Kidney Injury (PRAKI) in Western India
} Dr. Vipul Gattani MD, DM (Nephrology) ${ }^{1 *}$, Dr. Maulin K. Shah MD, DM (Nephrology) ${ }^{2}$

${ }^{1}$ Assistant Professor, Department of Medicine, Dr. Vasantrao Pawar Medical College, Hospital and Research Centre, Adgaon, Nashik, India

${ }^{2}$ Consultant Nephrologist and Transplant Physician, Assistant Professor, Department of Medicine, Shree Krishna Hospital, Karamsad, Anand, Gujrat, India

DOI: $10.36347 /$ sjams.2020.v08i05.015

| Received: 23.04.2020 | Accepted: 30.04.2020 | Published: 14.05.2020

*Corresponding author: Dr. Vipul Gattani MD DM

Abstract

Acute renal failure (ARF) of obstetric origin is one of the most life threatening complication of pregnancy which leads to poor foetal and maternal outcome. The incidence of pregnancy related acute kidney injury (PRAKI) in developed countries is 1 in 20000 pregnancies while in India, it is 1 in 50 pregnancies. Though data of PRAKI is available from previous studies, we have to study the prevalence on regular intervals so that appropriate health care measures could be taken immediately. Hence we aim to study the epidemiological and etiological factors of acute renal failure of obstetric origin in Indian society. Pregnant patients who were not having history of renal disease previously and developed acute renal failure as evident by oliguria ( urine output $<400 \mathrm{ml} / \mathrm{day}$ ) or rising azotaemia in presence of normal urine output were included in this study. Detailed history, clinical examination, investigation, management and follow up were done according to protocol. Out of total 1021 cases of acute renal failure, 96 (9.4\%) were of obstetric origin and enrolled in study as per inclusion criteria. The maximum incidence of PRAKI was found in the age group of 24-29 years (53.13\%) and it was least in patients above 35 years $(3.13 \%)$. We found puerperal sepsis as the most common etiological factor leading to PRAKI followed by Preeclampsia, Antepartum haemorrhage and Pregnancy related hemolytic uremic syndrome (HUS). The reason for decline in incidence of PRAKI could be the early and effective treatment of pregnancy related complications, improved healthcare facilities and improved health seeking behaviour of people. Health education, very well percolated prenatal care, safe and approved abortion services along with skilled delivery healthcare personnel were the factors that can be worked upon to reduce the epidemiological burden of PRAKI. Simultaneously, strengthening of referral and communication systems among primary, secondary and tertiary health care centres in India will definitely contribute in reducing maternal mortality due to PRAKI.

Keywords: Acute renal failure, Pregnancy related acute kidney injury.

Copyright @ 2020: This is an open-access article distributed under the terms of the Creative Commons Attribution license which permits unrestricted use, distribution, and reproduction in any medium for non-commercial use (NonCommercial, or CC-BY-NC) provided the original author and source are credited.

\section{INTRODUCTION}

Acute renal failure (ARF) of obstetric origin is one of the most life threatening complication of pregnancy which leads to poor foetal and maternal outcome. The incidence of pregnancy related acute kidney injury (PRAKI) in developed countries is 1 in 20000 pregnancies [1], whereas in developing countries like India, it is 1 in 50 pregnancies [2]. This marked decline in incidence of obstetric ARF in developed nations is attributed to near disappearance of postabortal sepsis and improved care of hypertension in pregnancy. Septic abortions, poor follow up of patient with pregnancy, limited screening of pregnant patient with hypertensive complications and late referral to specialized treatment centres are responsible for high incidence of obstetric ARF in developing nations [3].
Among all cases of ARF in developing countries, 15-20\% are PRAKI [4,5]. The prevalence of obstetric ARF among all cases of ARF decreased from $14.5 \%$ in the 1980 s to $7-10 \%$ in the $2010-15$ [6-11]. All the aetiologies that cause ARF in non-pregnant patient can cause ARF in pregnant patient like volume depletion, haemorrhage, sepsis in addition to pregnancy specific aetiologies like HELLP syndrome, acute fatty liver of pregnancy, thrombotic microangiopathy[3]. The causes of obstetric ARF can be divided into three groups based on the duration of pregnancy viz, first half, second half and postpartum ARF. Septic abortion is still the most common cause for ARF in first half, preeclampsia and antepartum haemorrhage (APH) are common in second half of pregnancy. Postpartum haemorrhage and puerperal sepsis are responsible for postpartum ARF. 
Epidemiological and etiological factors for ARF of obstetric origin differ as per the geographical region and over different time frame. Many of such cases are being referred to our tertiary care centre and mostly they belong to lower to middle socioeconomic class. Strengthening of peripheral primary and secondary care centres with good infrastructure, equipments and trained doctors may reduce the prevalence of pregnancy associated acute kidney injury in our country. At the same time, the problem statement under study has to be informed well to treating doctors and other stakeholders. Though data of PRAKI is available from previous studies, we have to study the prevalence on regular intervals so that appropriate health care measures could be taken immediately. And this continuous process of surveillance will help to improve the standard of health care in periphery. Hence, we aimed at evaluation of epidemiology and etiological factors of pregnancy related acute kidney injury [3].

\section{Aims \& OBJECTIVES}

To study epidemiological and etiological factors of acute renal failure of obstetric origin in Indian society.

\section{Material ANd Methods}

This was the prospective, observational study conducted at our government aided, tertiary care centre from January 2014 to December 2015.

Acute Kidney Injury (AKI) was defined on the basis of Risk, Injury, Failure, Loss of function, and End-stage renal disease (RIFLE) criteria [12]. PRAKI was defined as AKI diagnosed anytime during pregnancy or during postpartum phase (first 6 weeks post-delivery). During this study period, total 1021 patients were admitted to the hospital with acute renal failure due to various etiology. Out of which, 96 patients were due to renal failure from obstetric origin.

The study protocol was approved by the institutional ethics committee and included a detailed patient information brochure. Informed consent was obtained from all participants or their next of kin.
Pregnant patients who were not having history of renal disease previously and developed acute renal failure as evident by oliguria ( urine output $<400 \mathrm{ml} /$ day) or rising azotaemia in presence of normal urine output were included in this study. Such 96 enrolled patients were treated and prospectively studied with respect to their history, clinical features, laboratory parameters, precipitating factors for acute renal failure, its complications, maternal and foetal outcome, and renal outcome at 3 months of discharge.

Detailed history, clinical examination, investigation, management and follow up were done according to proforma of case report form. Each patient underwent complete obstetric examination and removal of products of conception was done as and when required. If the initial event leading to ARF began outside the hospital, the information was collected from the patient and her immediate attendants and via medical referral records and telephone calls to the referring doctors whenever possible. Details of obstetric, medical, and surgical managementincluding the need for blood transfusion, dialysis, and intensive care-were recorded. For haemodialysis, access was obtained either by femoral or right internal jugular vein.

All details were recorded in a structured format in Microsoft excel 2007. Continuous variables were expressed as mean+SD whereas categorical variables were expressed in absolute numbers or percentages.

\section{ObServation \& Results}

During our study period, total 1021 cases of acute renal failure were observed at our institute out of which $96(9.4 \%)$ were of obstetric origin and enrolled in study as per inclusion criteria. The mean age of patients with obstetric ARF in present study is 26.21 years. Youngest patient was 19 years old and eldest was 38 years old. The maximum incidence of obstetric ARF was found in the age group of 24-29 years $(53.13 \%)$ and it was least in patients above 35 years $(3.13 \%)$ (Table $1)$.

Table-1: Distribution of patients with obstetric ARF in different age groups

\begin{tabular}{|l|l|l|}
\hline Age distributions (in years) & No. of patients $(\mathbf{n = 9 6})$ & Percentage \\
\hline $18-23$ & 23 & $23.95 \%$ \\
\hline $24-29$ & 51 & $53.13 \%$ \\
\hline $30-35$ & 19 & $19.79 \%$ \\
\hline $36-38$ & 3 & $3.13 \%$ \\
\hline
\end{tabular}

We came across $27(28.13 \%)$ primigravida patients and $69(71.88 \%)$ patients as multigravida. In our study of 96 patients, 17 patients $(17.7 \%)$ presented in early pregnancy while 44 patients $(45.83 \%)$ presented in late pregnancy and 35 patients $(36.46 \%)$ presented in postpartum period (Table 2). Regarding registration for antenatal clinics, $67(69.79 \%)$ patients had taken antenatal care while $29(30.21 \%)$ patients had not received antenatal care. Out of all enrolled patients, $88(91.67 \%)$ had hospital delivery while $8(8.33 \%)$ had home delivery. 
Table-2: Pregnancy status at the time of presentation

\begin{tabular}{|l|l|l|l|}
\hline Sr. No. & Pregnancy status & No. of Patients & Percentage (\%) \\
\hline 1 & Early pregnancy $\left(1^{\mathrm{st}} \& 2^{\text {nd }}\right.$ trimester $)$ & 17 & 17.70 \\
\hline 2 & Late pregnancy $\left(3^{\mathrm{rd}}\right.$ trimester $)$ & 44 & 45.83 \\
\hline 3 & Postpartum & 35 & 36.46 \\
\hline
\end{tabular}

Mode of delivery for 30 patients $(31.25 \%)$ was caesarean section (LSCS) while rest $66(68.75 \%)$ had normal vaginal delivery. 24 pregnant patients required termination of pregnancy due to presence of ARF. Among them $37.5 \%$ patients had bleeding PV and $41.67 \%$ had IUD. Pregnancy was terminated in 4 patients with preeclampsia and 1 patient with foetal distress (Table 3). In present study of 96 patients, 70
(72.92\%) had oliguria, $21(21.88 \%)$ had anuria and 5 patients $(5.21 \%)$ had non oliguric renal failure on presentation. Patient presented with more than one symptom in addition to decrease or no urine output. Other than oliguria or anuria, common presenting symptoms were fever $(55.21 \%)$, bleeding per vaginal $(35.42 \%)$, breathlessness $(27.08 \%)$, oedema $(15.63 \%)$ (Table 4).

Table-3: Indication of induction and mode of delivery in pregnant patients with ARF reaching to hospital (our institute)

\begin{tabular}{|l|l|l|l|}
\hline Sr. No. & Reason for induction & Vaginal (18) & LSCS (6) \\
\hline 1 & Preeclampsia & 2 & 2 \\
\hline 2 & Bleeding PV & 8 & 1 \\
\hline 3 & IUD & 8 & 2 \\
\hline 4 & Foetal distress & - & 1 \\
\hline
\end{tabular}

Table-4: Symptoms and clinical parameters on presentation

\begin{tabular}{|l|l|l|l|}
\hline Sr. No. & Oymptoms & No. of Patients & Percentage (\%) \\
\hline 1 & Oliguria/Anuria/Non oliguric & $70 / 21 / 5$ & $72.92 / 21.88 / 5.21$ \\
\hline 2 & Fever & 53 & 55.21 \\
\hline 3 & Bleeding P/V & 34 & 35.42 \\
\hline 4 & Altered level of consciousness & 18 & 18.75 \\
\hline 5 & Convulsion & 9 & 9.38 \\
\hline 6 & Breathlessness & 26 & 27.08 \\
\hline 7 & Oedema & 15 & 15.63 \\
\hline 8 & Hypertension (BP > 140/90 mmHg or antihypertensive requirement) & 32 & 33.33 \\
\hline 9 & Hypotension (BP $<90 / 60 \mathrm{mmHg}$ or vasopressor requirement) & 11 & 11.46 \\
\hline
\end{tabular}

We encountered 27 (28.13\%) patients who had CNS dysfunction including altered level of consciousness and convulsion, 32 (33.33\%) had hypertension and $11(11.46 \%)$ had hypotension at presentation. We found puerperal sepsis in 61 of 96 patients $(63.54 \%)$ as the most common etiological factor leading to ARF in obstetrics patients.
Preeclampsia was second most common etiological factor in 22 patients $(22.92 \%)$ and had been complicated by presence of HELLP in 14 patients (14.58\%). Antepartum haemorrhage (APH) was observed in $21(21.88 \%)$ patients of obstetric ARF. Hemolytic uremic syndrome (HUS) was aetiology of ARF in 4 patients (Table 5).

Table-5: Various etiological factors for ARF of obstetric origin.

\begin{tabular}{|l|l|l|l|}
\hline Sr. No. & Etiological Factor & No. Of Patients & Percentage (\%) \\
\hline 1 & Post Abortion sepsis & 6 & 6.25 \\
\hline 2 & Haemorrhage (APH) & 21 & 21.88 \\
\hline 3 & Haemorrhage (PPH) & 15 & 15.63 \\
\hline 4 & Preeclampsia/Eclampsia & 22 & 22.92 \\
\hline 5 & Puerperal sepsis & 61 & 63.54 \\
\hline 6 & Malaria & 3 & 3.13 \\
\hline 7 & HELLP & 14 & 14.58 \\
\hline 8 & HUS & 4 & 4.17 \\
\hline 9 & Chronic kidney disease & 7 & 7.29 \\
\hline
\end{tabular}

\section{DisCUSSION}

Pregnancy-related acute renal failure is usually a consequence of obstetric complications, and therefore prevention measures should be directed at addressing the lacunae of existing maternity care $[13,14]$ 
The present investigation showed the incidence of obstetric ARF as 9.4 per cent. Although the incidence reported by various investigators in last decade in India ranges from $3.39 \%-9.82 \%[8-10,11,15-17]$ and even $22.1 \%$ in a study by Chugh et al. in 1976, it is evident that incidence of obstetric ARF has declined from
$22.1 \%$ in 1976 to $3.3 \%$ to $9.8 \%$ in 2015 [15]. The reason for decline could be due to the early and effective treatment of pregnancy related complications, improved healthcare facilities and improved health seeking behaviour of people (Table 6).

Table-6: Incidence of ARF of obstetric origin from different studies in India

\begin{tabular}{|l|l|l|l|}
\hline Author & Year of study & Region & Incidence of obstetric ARF \\
\hline Chugh et al.[15] & 1976 & North India & $22.1 \%$ \\
\hline Gopalakrishnan et al. $[9]$ & 2015 & South India & $7.8 \%$ \\
\hline Krishna et al. $[16]$ & 2015 & Lucknow & $3.39 \%$ \\
\hline Nazar et al. $[10]$ & 2008 & Kashmir valley & $7.02 \%$ \\
\hline Pahwa et al.[17] & 2014 & Indore & $3.59 \%$ \\
\hline Godara et al. $[11]$ & 2014 & West India & $9.82 \%$ \\
\hline Goplani et al. $[8]$ & 2008 & West India & $9.06 \%$ \\
\hline Present study & 2016 & West India & $9.4 \%$ \\
\hline
\end{tabular}

The mean age of patient in present study was 26.21 years which was in accordance with studies from West India by Pahwa et al. (26.7 years)[17], Godara et al. (26.4 years)[11], Goplani et al. (25.6 years) [8]. While it was higher in studies from North India by Krishna et al. (28.85\%)[16] and Nazar et al. $(28.94 \%)[10]$. This difference in region could be due to completion of fertility early due to lower age of marriage.

In the present study, $27(28.13 \%)$ patients were primigravida while $69(71.88 \%)$ patients were multigravida. This is in accordance with study by Najar et al. [10], where $30 \%$ cases were primigravida and $70 \%$ were multigravida while Krishna et al. [16] found $20.4 \%$ cases of primigravida and $79.6 \%$ of multigravida. Patients referred to our institute mostly belonged to low socioeconomic strata from rural areas where family structure involved more than one child and this could explain high number of multigravida in the present study.

Maximum patients in our study group were presented in late pregnancy. In a study by Krishna et al $15.3 \%$ of patients developed ARF in 1st trimester, $28.57 \%$ developed ARF in 2nd trimester while 56.12\% of patients were in 3rd trimester. In a study by Gopalkrishnan et al. most of the patients (68\%) presented in postpartum period [9]. In contrast to our observations, earlier studies by Chugh et al. in 1976 of 72 patients, 43 patients $(59.7 \%)$ developed ARF in early pregnancy while $29(40.35 \%)$ patients developed ARF in late pregnancy [15]. This difference could be due to high number of ARF due to abortion during those days.

Out of 96 patients studied, $67(69.79 \%)$ patients had taken antenatal care while 29 (30.21\%) patients had not received antenatal care. Similar findings were observed by Krishna et al. where 63 of 98 patients $(64.28 \%)$ had antenatal care while $35(35.71 \%)$ patients did not receive antenatal care [16]. However, the lack of prenatal care in India is more than a medical problem because associated sociocultural, economic, and geographic barriers often prevent women from receiving appropriate obstetric care [18]. Very few of our study patients had delivered at home. This is in accordance with the study by Goplani et al. 59 of 70 patients $(84.28 \%)$ had hospital delivery and 11 patients $(15.71 \%)$ had home delivery [8].

In present study out of 96 patients 70 patients (72.92\%) had oliguria, 21 patients $(21.88 \%)$ had anuria and 5 patients $(5.21 \%)$ had non oliguric renal failure on presentation. Similar findings were observed in a study by Godara et al where $64.9 \%$ patients were oliguric and $21 \%$ were anuric [11]. Patient presented with more than one symptom in addition to decrease or no urine output. Other than oliguria or anuria, common presenting symptoms were fever $(55.21 \%)$, bleeding per vaginal $(35.42 \%)$, breathlessness $(27.08 \%)$, oedema $(15.63 \%)$. There were fewer incidences of breathlessness and oedema in present study as compared to that seen in study by Nazar et al. [10], where it occurred in $50 \%$ and $60 \%$ respectively. This difference was due to the haemodialysis which patients have already received before they were referred to our centre.

As compared to earlier study by Chugh et al. in 1976 where postabortion sepsis accounted for $59.7 \%$ of cases [15], as against $6.25 \%$ in present study. There is decline in incidence of postabortion sepsis which is also observed by other investigators from different parts of the country. Although alarming, the relative contribution of ARF following abortion was still lower than in other Indian studies [13, 14], which may be because of the legalization of abortion, more public awareness about the complications of illegal abortion, and more recently the availability of better reproductive healthcare facilities - especially through the National Rural Health Mission in India [19]. Its incidence could be reduced further by preventing unplanned and unwanted pregnancies through increased use of regular contraception, backed-up by emergency methods [20]. Similarly, there has been a decrease in the incidence of 
ARF related to puerperal sepsis [13], which occurred in $9(17.8 \%)$ cases in the present study. Increased numbers of deliveries by skilled birth attendants and availability of potent antibiotics have led to a reduction of more than $50 \%$ in the incidence of ARF due to puerperal sepsis [21].

Similarly, in a study by Gopalakrishnan et al. sepsis $(39 \%)$ was the most common aetiology for obstetric ARF followed by preeclampsia (21\%), antepartum haemorrhage $(10 \%)$ and thrombotic microangiopathy (9\%)[9].
In the present study, 7 patients of 96 (7.29\%) presented with acute renal failure indeed had chronic kidney disease as evident by bilateral small sized kidneys on ultrasonography. These patients were completely asymptomatic before conception and ARF in pregnancy could be the first presentation of chronic kidney disease. 5 of 7 patients presented early in the pregnancy and did not recover the renal function while 2 patients who presented late near term had partial recovery. Other investigators have excluded patients with structural kidney diseases from the study.

Table-7: Comparison of etiological factors from studies by other investigators from different parts of country

\begin{tabular}{|c|l|c|c|c|c|}
\hline $\begin{array}{c}\text { Sr. } \\
\text { No. }\end{array}$ & $\begin{array}{l}\text { Etiological } \\
\text { Factor }\end{array}$ & $\begin{array}{c}\text { Present } \\
\text { study }\end{array}$ & $\begin{array}{c}\text { Nazar } \text { et al. } \\
{[\mathbf{1 2} \text { (Kashmir }} \\
\text { Valley) }\end{array}$ & $\begin{array}{c}\text { Krishna } \text { et } \\
\text { al. } \text { [6] } \\
\text { (Lukhnow) }\end{array}$ & $\begin{array}{c}\text { Gopalkrishnan } \\
\text { et al. } \text { [11] } \\
\text { (South India) }\end{array}$ \\
\hline 1 & Post Abortion sepsis & 6.25 & 50 & 32.65 & - \\
\hline 2 & Haemorrhage (APH) & 21.88 & 15 & 23.46 a & 10 \\
\hline 3 & Haemorrhage (PPH) & 15.63 & 5 & - & 2 \\
\hline 4 & Preeclampsia/eclampsia & 22.92 & 15 & 14.28 & 21 \\
\hline 5 & Puerperal sepsis & 63.54 & NA & 23.46 & 39 b \\
\hline 6 & HUS & 4.17 & 2.5 & 2.04 & 9 \\
\hline 7 & CKD & 7.29 & - & - & - \\
\hline
\end{tabular}

From above comparison it is evident that etiological factors differ as per the region. Postabortion sepsis was still a common cause for ARF in studies by Nazar et al. [10] and Krishna et al. [16] while haemorrhage was much more common in present study as compared to others. In present study some patients had more than one etiological factor responsible for ARF.

Pre-eclampsia/eclampsia remains a major cause of PRARF. In southern India, the most common cause of PRARF has changed from hemorrhage to hypertensive disorders over the past 20 years [22]. In the present study, hypertensive disorders contributed to approximately one-quarter of PRARF cases. The fact that eclampsia accounted for most $(53.3 \%)$ of the hypertensive disorders indicates that this condition was not adequately managed in its initial stages. Considering the report of 2005-2006 National Family Health Survey [23], only 50\% of pregnant women in India had at least 3 prenatal check-ups. Incomplete coverage of prenatal care could be an important underlying factor in these complications being missed in their initial stages. Therefore, concerted efforts are required to improve the coverage of prenatal care, especially in peripheral areas.

Hemorrhage leading to hypotension has been implicated as an important causative factor in PRARF [24]. In a recent study from Pakistan, hemorrhage was the most common cause of PRARF [25], and it was the second most common cause in the present study. All hemorrhage-related case fatality occurred following caesarean delivery, which again highlights the need to evaluate the safety of this form of delivery at peripheral hospitals in low-income countries. However, early and prompt volume replacement at the first referral unit and quick access to high-quality emergency obstetric careincluding adequate blood transfusion services-would help to reduce the consequences of hemorrhage and incidence of PRARF [21, 24]. However, difficult road systems and a lack of adequate facilities often add to the burden of this life-threatening situation [26].

\section{Conclusion}

Sepsis, hemorrhage, and hypertensive disorders of pregnancy were the most important etiological factors for pregnancy related acute renal failure. Health education, very well percolated prenatal care, safe and approved abortion services along with skilled delivery healthcare personnel were the factors that can be worked upon to reduce the epidemiological burden of PRARF. Simultaneously, strengthening of referral and communication systems among primary, secondary and tertiary health care centres in India will definitely contribute in reducing maternal mortality due to PRARF.

\section{REFERENCES}

1. Stratta P, Besso L, Canavese C, Grill A, Todros T, Benedetto C, Hollo S, Segoloni GP: Is pregnancyrelated acute renal failure a disappearing clinical entity? Ren Fail. 18: 575-584, 1996.

2. Prakash J, Niwas SS, Parekh A, Pandey LK, Sharatchandra L, Arora P, Mahapatra AK: Acute kidney injury in late pregnancy in developing countries. Ren Fail. 32: 309-313, 2010 
3. Fakhouri F, Vercel C, Fre'meaux-Bacchi V. Obstetric Nephrology: AKI and Thrombotic Microangiopathies in Pregnancy Clin J Am Soc Nephrol. 7: 2100-2106.

4. Selcuk NY, Onbul HZ, San A, Odabas AR. Changes in frequency and etiology of acute renal failure in pregnancy (1980-1997) Ren Fail. 1998;20:513-7

5. Naqvi R, Akthar F, Ahmad E, Shaikh R, Ahmed Z, Naqvi A. Acute renal failure of obstetrical origin during 1994 at one centre. Ren Fail. 1996;18:6813.

6. Chugh KS. Etiopathogenesis of acute renal failure in the tropics. Ann Natl Acad Med Sci (India). 1987;3:88-99.

7. Kilari SK, Chinta RK, Vishnubhotla SK. Pregnancy related acute renal failure. J Obstet Gynecol India.2006;56:308-10.

8. Goplani KR, Shah PR, Gera DN, Gumber M, Dabhi M, Feroz A. Pregnancy-related acute renal failure: A single-center experience. Indian $\mathbf{J}$ Nephrol. 2008 Jan;18(1):17-21.

9. Gopalakrishnan N, Dhanapriya J, Muthukumar P, Sakthirajan R, Dineshkumar T, Thirumurugan s et al Acute kidney injury in pregnancy--a single center experience. Ren Fail. 2015;37(9):1476-80.

10. Nazar MS, Shah AR, Wani IA, Reshi R, Banday KA, Bhat MA. Pregnancy related acute kidney injury: A single center experience from the Kashmir Valley. Indian J Nephrol. 2008 Oct; 18(4): 159-161.

11. Godara SM, Kute VB, Trivedi HL, Vanikar AV, Shah PR, Gumber MR. Clinical profile and outcome of acute kidney injury related to pregnancy in developing countries: a single-center study from India. Saudi J Kidney Dis Transpl. 2014 Jul;25(4):906-11.

12. Mahesh E, Puri S, Varma V, Madhyastha PR, Bande S, Gurudev KC. Pregnancy-related acute kidney injury: An analysis of 165 cases. Indian J Nephrol. 2017;27:113-7.

13. Prakash J, Kumar H, Sinha DK, Kedalaya PG, Pandey LK, Srivastava PK. Acute renal failure in pregnancy in a developing country: twenty years of experience. Ren Fail. 2006;28(4):309-13.

14. Goplani KR, Shah PR, Gera DN, GumberM, DabhiM, Feroz A. Pregnancy-related acute renal failure: a single-center experience. Indian $\mathrm{J}$ Nephrol. 2008;18(1):17-21.

15. Chugh KS, Singhal PC, Kher VK, Gupta VK, Malik GH, Narayan G, Datta BN. Spectrum of acute cortical necrosis in Indian patients. Am J Med Sci. 1983; 286: 10-20.

16. Krishna A, Singh R, Prasad N, Gupta A, Bhadauria D, Kaul A. Maternal, fetal and renal outcomes of pregnancy - associated acute kidney injury requiring dialysis. Ind J Nephrol. 25(2):77-81.

17. Pahwa N, Bharani R, Kumar R. Post-partum acute kidney injury. Saudi J Kidney Dis Transpl. 2014 Nov;25(6):1244-7.

18. Ministry of Health and Family Welfare, Government of India. Family Welfare Statiatics in India-2006. New Delhi, India: Ministry of Health and Family Welfare, Government of India; 2007.

19. Sharma AK. National rural health mission: time to take stock. Indian J Community Med. 2009;34(3):175-82.

20. Arora N, Mittal S. Emergency contraception and prevention of induced abortion in India. J Fam Plan Reprod Health Care. 2005;31(4):294-6.

21. Majhi AK. Emergency obstetric care-present focuses to reduce maternal mortality. Indian $\mathbf{J}$ Perinatol Reprod Biol. 2006;18(1):11-3.

22. Rani PU, Narayen G, Anuradha. Changing trends in pregnancy related acute renal failure. J Obstet Gynecol India. 2002;52(1):36-8.

23. International Institute for Population Sciences (IIPS) and ORC Marco. National Family Health Survey (NFHS-3), India, 2005-2006. Mumbai, India: IIPS; 2007.

24. Kongnyuy EJ, Hofman JJ, van den Broek N. Ensuring effective Essential Obstetric Care in resource poor settings. BJOG Oct. 2009;116(Suppl $1): 41-7$.

25. Ansari MR, Laghari MS, Solangi BK. Acute renal failure in pregnancy: one year observational study at Liaquat. J Pakistan Med Assoc. 2008;58(2):614.

26. Biswas AB, Nandy S, Sinha RN, Das DK, Roy RN, Datta S. Status of maternal and new born care at first referral units in the state of West Bengal. Indian J Public Health. 2004;48(1):21-6. 